\title{
reciam muc
}

Revista cientifica de investigación actualización del mundo de las ciencias

Daniel Fernando Dick Paredes ${ }^{\text {a; }}$ Jenniffer Cristina Pazmiño Farfán ${ }^{\text {b; }}$ John Michael

Franco Orellana ${ }^{c}$; Mayra Alexandra Bermeo Naranjo ${ }^{d}$

Epilepsia infantil diagnóstico, tratamiento y recomendación para padres

Childhood epilepsy diagnosis, treatment and recommendation for parents

Revista Científica de Investigación actualización del mundo de las Ciencias. Vol. 3 núm., 1, enero, ISSN: 2588-0748, 2018, pp. 147-163

DOI: $10.26820 /$ reciamuc/3.(1).enero.2019.147-163

URL: http://reciamuc.com/index.php/RECIAMUC/article/view/227

Código UNESCO: 3205 Medicina Interna

Tipo de Investigación: Artículo de Revisión

Editorial Saberes del Conocimiento

Recibido: 10/12/2018

Aceptado: 20/01/2019

Publicado: 30/01/2019

Correspondencia: director@ reciamuc.com

a. Médico; Guayaquil, Ecuador.
b. Médico; Guayaquil, Ecuador.
c. Médico; Guayaquil, Ecuador.
d. Médico; Guayaquil, Ecuador. 


\section{Epilepsia infantil diagnóstico, tratamiento y recomendación para padres}

Vol. 3, núm. 1., (2019)

Daniel Fernando Dick Paredes; Jenniffer Cristina Pazmiño Farfán; John Michael Franco Orellana; Mayra Alexandra Bermeo Naranjo

\section{RESUMEN}

En los últimos años hay una rápida expansión en nuestra comprensión de la epilepsia. El desarrollo de nuevos fármacos antiepilépticos y los refinamientos de la cirugía de epilepsia están ampliando las opciones terapéuticas para la epilepsia. Además, la clasificación de las epilepsias continúa evolucionando en función de una mayor comprensión de la genética molecular de la enfermedad y esto incluye el reconocimiento de posibles nuevos síndromes de epilepsia. Esta revisión considera algunos de estos desarrollos interesantes, además de abordar las características esenciales del diagnóstico, las investigaciones, manejo e impacto de la epilepsia en la infancia.

Palabras Claves: Niños; Epilepsia; Convulsiones; Medicamentos Antiepilépticos. 


\title{
Epilepsia infantil diagnóstico, tratamiento y recomendación para padres
}

Vol. 3, núm. 1., (2019)

Daniel Fernando Dick Paredes; Jenniffer Cristina Pazmiño Farfán; John Michael Franco

Orellana; Mayra Alexandra Bermeo Naranjo

\begin{abstract}
In recent years there is a rapid expansion in our understanding of epilepsy. The development of new antiepileptic drugs and the refinements of epilepsy surgery are expanding the therapeutic options for epilepsy. In addition, the classification of epilepsies continues to evolve based on a greater understanding of the molecular genetics of the disease and this includes the recognition of possible new epilepsy syndromes. This review considers some of these interesting developments, in addition to addressing the essential characteristics of the diagnosis, research, management and impact of epilepsy in childhood.
\end{abstract}

Key Words: Children; Epilepsy; Convulsions; Antiepileptic drugs. 


\section{Epilepsia infantil diagnóstico, tratamiento y recomendación para padres}

Vol. 3, núm. 1., (2019)

Daniel Fernando Dick Paredes; Jenniffer Cristina Pazmiño Farfán; John Michael Franco Orellana; Mayra Alexandra Bermeo Naranjo

\section{Introducción.}

La epilepsia es un problema neurológico heterogéneo común en los niños. Ejerce un importante peaje físico, psicológico, económico y social en niños y sus cuidadores. Cincuenta millones de personas tienen epilepsias globalmente, más de la mitad de ellos son niños. Solo en Estados Unidos entre 25.000 y 40.000 niños tendrán una primera convulsión no febril cada año. El problema se complica aún más en los países en desarrollo, ya que añaden alrededor del 7580\% de los nuevos casos de epilepsia (Guerrini, 2006).

Las convulsiones y las epilepsias en los niños son extremadamente diversas, que difieren notablemente en la edad de inicio, convulsiones características, comorbilidades asociadas, tratamiento y pronóstico. Dado que hay una escasez de pediatras epileptólogos que practican en todo el mundo, es imposible para todos los niños con convulsiones recurrentes a recibir su atención de subespecialistas. Más bien, los niños a menudo necesitan confiar en neurólogos pediátricos, pediatras en general y / o médicos de familia que le proporcionen la atención que necesitan y merecen. Sin una firme comprensión de las complejidades de la epilepsia en la infancia, tal vez no sea posible para tales médicos hacer un diagnóstico preciso y planee una estrategia de tratamiento eficaz. Por eso es importante para el pediatra general estar al tanto de la evaluación y gestión de estos pacientes (Tamber \& Mountz, 2012). Además, pediatras y padres deben tener un papel en la prevención de la epilepsia minimizando los riesgos neurológicos en los primeros años de la infancia. Además, hay una necesidad de centrarse en proveedores de atención primaria de salud proporcionándoles pautas para prácticas óptimas, terapia racional y asesoramiento. 


\section{Epilepsia infantil diagnóstico, tratamiento y recomendación para padres}

Vol. 3, núm. 1., (2019)

Daniel Fernando Dick Paredes; Jenniffer Cristina Pazmiño Farfán; John Michael Franco

Orellana; Mayra Alexandra Bermeo Naranjo

\section{Metodología.}

Para el desarrollo de este proceso investigativo, se plantea como metodología la encaminada hacia una orientación científica particular que se encuentra determinada por la necesidad de indagar en forma precisa y coherente una situación, en tal sentido (Davila, 2015) define la metodología "como aquellos pasos previos que son seleccionados por el investigador para lograr resultados favorables que le ayuden a plantear nuevas ideas". (p.66)

Lo citado por el autor, lleva a entender que el desarrollo de la acción investigativa busca simplemente coordinar acciones enmarcadas en una revisión bibliográfica con el fin de complementar ideas previas relacionadas a las características, tratamiento y prevención de la epilepsia infantil a través de una revisión de literatura, para así finalmente elaborar un cuerpo de consideraciones generales que ayuden a ampliar el interés propuesto.

\section{Tipo de Investigación}

Dentro de toda práctica investigativa, se precisan acciones de carácter metodológico mediante las cuales, se logra conocer y proyectar los eventos posibles que la determinan, así como las características que hacen del acto científico un proceso interactivo ajustado a una realidad posible de ser interpretada. En este sentido, se puede decir, que la presente investigación corresponde al tipo documental, definido por Castro (2016), "se ocupa del estudio de problemas planteados a nivel teórico, la información requerida para abordarlos se encuentra básicamente en materiales impresos, audiovisuales y /o electrónicos”. (p.41). 


\section{Epilepsia infantil diagnóstico, tratamiento y recomendación para padres}

Vol. 3, núm. 1., (2019)

Daniel Fernando Dick Paredes; Jenniffer Cristina Pazmiño Farfán; John Michael Franco Orellana; Mayra Alexandra Bermeo Naranjo

En consideración a esta definición, la orientación metodológica permitió la oportunidad de cumplir con una serie de actividades inherentes a la revisión y lectura de diversos documentos donde se encontraron ideas explicitas relacionadas con los tópicos encargados de identificar a cada característica insertada en el estudio. Por lo tanto, se realizaron continuas interpretaciones con el claro propósito de revisar aquellas apreciaciones o investigaciones propuestas por diferentes investigadores relacionadas con el tema de interés, para luego dar la respectiva argumentación a los planteamientos, en función a las necesidades encontradas en la indagación.

\section{Fuentes Documentales}

El análisis correspondiente a las características que predomina en el tema seleccionado, llevan a incluir diferentes fuentes documentales encargadas de darle el respectivo apoyo y en ese sentido cumplir con la valoración de los hechos a fin de generar nuevos criterios que sirven de referencia a otros procesos investigativos. Para (CASTRO, 2016) las fuentes documentales incorporadas en la investigación documental o bibliográfica, "representa la suma de materiales sistemáticos que son revisados en forma rigurosa y profunda para llegar a un análisis del fenómeno".(p.41). Por lo tanto, se procedió a cumplir con la realización de una lectura previa determinada para encontrar aquellos aspectos estrechamente vinculados con el tema, con el fin de explicar mediante un desarrollo las respectivas apreciaciones generales de importancia.

\section{Técnicas para la Recolección de la Información}

La conducción de la investigación para ser realizada en función a las particularidades que determinan a los estudios documentales, tiene como fin el desarrollo de un conjunto de acciones encargadas de llevar a la selección de técnicas estrechamente vinculadas con las características 


\section{Epilepsia infantil diagnóstico, tratamiento y recomendación para padres}

Vol. 3, núm. 1., (2019)

Daniel Fernando Dick Paredes; Jenniffer Cristina Pazmiño Farfán; John Michael Franco Orellana; Mayra Alexandra Bermeo Naranjo

del estudio. En tal sentido, (Bolívar, 2015), refiere, que es "una técnica particular para aportar ayuda a los procedimientos de selección de las ideas primarias y secundarias”. (p. 71).

Por ello, se procedió a la utilización del subrayado, resúmenes, fichaje, como parte básica para la revisión y selección de los documentos que presentan el contenido teórico. Es decir, que mediante la aplicación de estas técnicas se pudo llegar a recoger informaciones en cuanto a la revisión bibliográfica de los diversos elementos encargados de orientar el proceso de investigación. Tal como lo expresa, (Bolívar, 2015) "las técnicas documentales proporcionan las herramientas esenciales y determinantes para responder a los objetivos formulados y llegar a resultados efectivos" (p. 58). Es decir, para responder con eficiencia a las necesidades investigativas, se introdujeron como técnica de recolección el método inductivo, que hizo posible llevar a cabo una valoración de los hechos de forma particular para llegar a la explicación desde una visión general.

Asimismo, se emplearon las técnicas de análisis de información para la realización de la investigación que fue ejecutada bajo la dinámica de aplicar diversos elementos encargados de determinar el camino a recorrer por el estudio, según, (Bolívar, 2015) las técnicas de procesamiento de datos en los estudios documentales "son las encargadas de ofrecer al investigador la visión o pasos que debe cumplir durante su ejercicio, cada una de ellas debe estar en correspondencia con el nivel a emplear" (p. 123). Esto indica, que para llevar a cabo el procesamiento de los datos obtenidos una vez aplicado las técnicas seleccionadas, tales como: fichas de resumen, textual, registros descriptivos entre otros, los mismos se deben ajustar al nivel que ha sido seleccionado. 


\section{Epilepsia infantil diagnóstico, tratamiento y recomendación para padres}

Vol. 3, núm. 1., (2019)

Daniel Fernando Dick Paredes; Jenniffer Cristina Pazmiño Farfán; John Michael Franco Orellana; Mayra Alexandra Bermeo Naranjo

\section{Resultados.}

Una convulsión se define como una explosión excesiva y anormal de actividad neuronal sincronizada que afecta a pequeños o grandes redes neuronales que dan lugar a manifestaciones clínicas que son repentinas, transitorias y usualmente breves. Según (Tamber \& Mountz, 2012) la epilepsia es definida como un trastorno del cerebro caracterizado por cualquiera de las siguientes condiciones:

1. Al menos dos convulsiones no provocadas (o reflejos) que ocurren $>24 \mathrm{~h}$ de diferencia.

2. Una convulsión no provocada (o reflejo) y una probabilidad mayor de convulsiones similares al riesgo general de recurrencia (al menos 60\%) después de dos convulsiones no provocadas, ocurridas durante los próximos 10 años.

3. Diagnóstico de un síndrome de epilepsia.

La convulsión provocada es una convulsión que ocurre en reacción a una condición aguda y transitoria afectando el cerebro. Los factores provocadores incluyen, pero no son limitado a, traumatismo craneal, accidente cerebrovascular, infecciones intracraneales, trastornos metabólicos agudos (por ejemplo, hipoglucemia, anoxia) y envenenamiento agudo por drogas o toxinas (Hauser \& Banerjee, 2008).

\section{Incidencia}

Las crisis epilépticas afectan al 1-2\% de la población y $4 \%$ de los niños. Los países en desarrollo tienen mayor prevalencia debida a la atención y estándares perinatales más pobres de 


\section{Epilepsia infantil diagnóstico, tratamiento y recomendación para padres}

Vol. 3, núm. 1., (2019)

Daniel Fernando Dick Paredes; Jenniffer Cristina Pazmiño Farfán; John Michael Franco

Orellana; Mayra Alexandra Bermeo Naranjo

nutrición e higiene pública y el mayor riesgo de lesión cerebral, infección cerebral u otro síntoma. La incidencia de convulsiones es la edad dependiente. La tasa de incidencia más alta (100 por 100,000) se observa en el primer año de vida, declinando a aproximadamente 20 casos por 100,000 por año en la adolescencia. La epilepsia infantil tiene una prevalencia de aproximadamente $0.5-0.8 \%$ y comprende un grupo heterogéneo de trastornos, incluyendo una variedad de síndromes de epilepsia que varían en gravedad de benigno a progresivo y catastrófico.

Hay una condición estructural distinta o enfermedad metabolica que se ha demostrado estar asociada con un mayor riesgo de desarrollar epilepsia. Las lesiones estructurales incluyen trastornos adquiridos tales como accidente cerebrovascular, traumatismo e infección. También pueden ser de origen genético (por ejemplo, esclerosis tuberosa).

La naturaleza de la causa subyacente es todavía desconocida; puede tener una base genética (por ejemplo, una canalopatía no reconocida) o puede ser el resultado de un trastorno estructural o metabólico no reconocido. Las epilepsias de causa desconocida son aquellas que en el pasado fueron denominadas "criptogénicos".

\section{Síndromes epilépticos en niños}

Se ha reportado que este síndrome ocurre en 14.4 por 100.000 nacidos vivos. La epilepsia neonatal familiar benigna predominantemente hereditario es debido a mutaciones que afectan el potasio dependiente de voltaje genes de canal (KCNQ2, KCNQ3). Los infantes afectados son generalmente a término y aparecen normales al nacer. En el $80 \%$ de los casos, las convulsiones comienzan el segundo o tercer día de vida, aunque algunos bebés pueden desarrollar 


\section{Epilepsia infantil diagnóstico, tratamiento y recomendación para padres}

Vol. 3, núm. 1., (2019)

Daniel Fernando Dick Paredes; Jenniffer Cristina Pazmiño Farfán; John Michael Franco Orellana; Mayra Alexandra Bermeo Naranjo

convulsiones más tarde en el primer mes de vida. Las convulsiones son típicamente clónicas, pero a menudo precedido por un componente tónico. Ellos son más a menudo unilaterales, pero también pueden ser bilaterales. El interictal EEG suele ser normal. La resolución espontánea típicamente ocurre dentro de 2 a 6 meses. Hay un ligero aumento en el riesgo de epilepsia posterior (11-15\%) (Berg, 2011).

Manejo de la epilepsia infantil

\section{Diagnóstico y diagnóstico diferencial}

Las tareas de diagnóstico en el manejo de la epilepsia incluyen un diagnóstico de convulsiones y un estudio etiológico estableciendo el diagnóstico e identificación de factores precipitantes. Esto se logra mediante una combinación de historia, examen físico, electroencefalografía (EEG) y exámenes de laboratorio (Piña-Garza, 2013)

\section{Diagnóstico de convulsiones}

El primer paso en el manejo de un niño para quien un diagnóstico de la epilepsia es posible, es establecer definitivamente si el paciente tiene epilepsia o no. Pacientes diagnosticados erróneamente de epilepsia será sometido innecesariamente a muchos medicamentos que pueden producir efectos secundarios graves.

Las entidades diferenciales específicas de diagnóstico que deben diferenciarse de las convulsiones incluyen las condiciones que imitan la epilepsia; confirmando o descartando la epilepsia no solo previene el tratamiento innecesario y la exposición a intervenciones, sino también reduce la ansiedad familiar y del paciente. 


\section{Epilepsia infantil diagnóstico, tratamiento y recomendación para padres}

Vol. 3, núm. 1., (2019)

Daniel Fernando Dick Paredes; Jenniffer Cristina Pazmiño Farfán; John Michael Franco Orellana; Mayra Alexandra Bermeo Naranjo

La mejor manera de diagnosticar qué tipo de convulsión sufre el paciente se debe observar una convulsión, aunque el médico por lo general no tiene la oportunidad de hacerlo. A menudo, la información de diagnóstico diferencial más importante es contenida en la historia recogida del paciente. El diagnóstico debe incluir detalles exactos de eventos antes, durante y después de la convulsión, obtenida del paciente y observadores. Síntomas y signos de convulsiones focales (motor, sensorial, autonómica, psíquica); alteración de conciencia; automatismos movimientos tónicos, movimientos clónicos, o ambos; morderse la lengua, incontinencia y el comportamiento postictal son detalles importantes. La duración, momento de ocurrencia (por ejemplo, al despertar, cuando está somnoliento, durante el sueño) y la frecuencia de las convulsiones también son importantes. Por ejemplo, convulsiones tónico-clónicas que ocurren durante las primeras horas de sueño suelen ser generalizadas secundarias, mientras que las convulsiones tónico-clónicas se producen sobre el despertar suele ser principalmente ocurrencia actual de otros tipos de convulsiones (especialmente mioclónica).

La historia para la etiología debe incluir preguntas con respecto a los antecedentes familiares de epilepsia, traumatismo craneal, parto, complicaciones, convulsiones febriles, oído medio e infecciones sinusales (que pueden erosionar a través de los huesos y causa foco cerebral) y síntomas de malignidad. La historia de los factores precipitantes debe incluir factores como fiebre, ansiedad, falta de sueño, hiperventilación, luces parpadeantes, o televisión.

\section{Examen físico}

Examen clínico, neurológico y de fondo son muy importantes en la evaluación de epilepsia. El examen físico debe ser dirigido hacia el descubrimiento de evidencia de trauma de 


\section{Epilepsia infantil diagnóstico, tratamiento y recomendación para padres}

Vol. 3, núm. 1., (2019)

Daniel Fernando Dick Paredes; Jenniffer Cristina Pazmiño Farfán; John Michael Franco Orellana; Mayra Alexandra Bermeo Naranjo

cabeza pasada o reciente; infecciones de las orejas y los senos paranasales; anomalías congénitas (por ejemplo, hemiatrofia, estigmas de tuberosa esclerosis); anomalías neurológicas focales o difusas y signos de malignidad. Otros hallazgos pueden ser útiles en el descubrimiento de evidencia de disfunción cerebral focal indicativo de epilepsia focal, por ejemplo, asimetría facial, asimetría del tamaño del pulgar (indica daño cerebral contralateral durante la infancia), pronación de manos extendidas, posturas distónicas cuando camina sobre los lados de los pies.

\section{Investigaciones}

Alrededor del $80 \%$ de la epilepsia comunitaria puede ser gestionada sin ninguna investigación. Estos no son para hacer un diagnóstico de epilepsia, pero si para confirmar, diagnosticar y establecer la causa. Pantalla metabólica y punción lumbar (por presión de apertura, recuentos celulares, proteínas, glucosa, citología, cultivo y serología) debe realizarse en caso de infección o sospecha de malignidad.

\section{Electroencefalografía}

El EEG es una herramienta de diagnóstico útil en la investigación de un trastorno convulsivo. Confirma la presencia de actividad eléctrica anormal dando información sobre el tipo de trastorno convulsivo y revela la ubicación del foco de ataque. Ambas rutinas (Rastreo de papel) y técnicas de grabación digital están en uso regular. El EEG realizado a las 24 h de la crisis se ha encontrado que tienen más rendimientos de descargas epileptiformes en comparación con un EEG hecho más tarde, 51 versus 34\%. El EEG normal no excluye la epilepsia, porque el 40\% de los pacientes epilépticos tienen un EEG normal. El monitoreo de EEG está reservado para casos complicados de convulsiones prolongadas y que no responden (Sharma, 2013) 


\section{Epilepsia infantil diagnóstico, tratamiento y recomendación para padres}

Vol. 3, núm. 1., (2019)

Daniel Fernando Dick Paredes; Jenniffer Cristina Pazmiño Farfán; John Michael Franco

Orellana; Mayra Alexandra Bermeo Naranjo

\section{Radiología}

Neuro-imágenes deben realizarse cuando un proveedor de atención médica sospecha una lesión cerebral estructural grave en pacientes con nuevos déficits focales, estado mental alterado persistentemente, fiebre, trauma reciente, cefalea persistente, historia de cáncer, historia de anticoagulación, o sospecha de síndrome de inmunodeficiencia adquirida.

\section{Síntesis de datos}

Combinando historia, examen físico e información de EEG, el proveedor de atención médica debe ser capaz de determinar (a) si los eventos del paciente son convulsiones o no, y (b) tipo de convulsiones del paciente. Si esto no se puede hacer, historial adicional (por ejemplo, testigos adicionales) o EEG adicional (por ejemplo, información de monitoreo de EEG a largo plazo), o ambos, deben ser adquirido. Si toda la información posible ha sido reunida y el diagnóstico sigue siendo incierto, el medico generalmente se ve obligado a actuar sobre la base de historia disponible. Si la historia sugiere fuertemente un tipo de crisis recurrente, un ensayo terapéutico de medicación antiepiléptica apropiada para la convulsión. Si la historia no hace sugerir convulsiones recurrentes, observación sin la medicación es el plan habitual. Tipo de convulsión combinada con información adicional de la historia, examen físico, EEG y pruebas de laboratorio a menudo permiten determinación de la epilepsia específica del paciente. Esta determinación ayuda con la selección de terapia y asesoramiento sobre el pronóstico y aparición familiar. 


\section{Epilepsia infantil diagnóstico, tratamiento y recomendación para padres}

Vol. 3, núm. 1., (2019)

Daniel Fernando Dick Paredes; Jenniffer Cristina Pazmiño Farfán; John Michael Franco Orellana; Mayra Alexandra Bermeo Naranjo

\section{Prevención}

Ha habido poca investigación y poca discusión dentro de la literatura sobre este potencialmente importante aspecto de la epilepsia. En vista del hecho de que una significativa proporción de epilepsia en la infancia (hasta 30-40\%, posiblemente más alto) tiene una base genética y dado que para la mayoría de estos pacientes genéticos "modificación" nunca es practicable o clínicamente justificado (independientemente de la dimensión ética), es poco probable que muchos de estas epilepsias siempre serán "prevenibles". Por mas de una decada, se ha afirmado que con una mayor comprensión de las bases moleculares (fisiopatológicas y genéticas) de la epilepsia, incluyendo los relativamente recientes descubiertos canalopatías, habría tratamientos novedosos y dirigidos para las epilepsias. Lamentablemente y no inesperadamente, esto no tiene, y es poco probable que se convierta en una realidad incluso dentro de la siguiente década. Obviamente, donde la epilepsia es una enfermedad común y la incapacitación de la manifestación de una genética y trastorno neurodegenerativo, como el ceroide neuronal, lipofuscinosis, modificación genética y / o terapia puede ser justificable, aunque todavía no necesariamente factible. Sin embargo, hay muchas situaciones donde la prevención de la epilepsia o consecuencias de la epilepsia será deseable y alcanzable; esto es particularmente aplicable, pero ciertamente no exclusivamente, en el mundo en desarrollo:

- Reducción de la incidencia de alto riesgo, incluyendo adolescentes embarazos.

- Mejor seguimiento y atención prenatal de las mujeres con embarazos de alto riesgo.

- Mejora de la atención perinatal y prevención de cualquier daño cerebral secundario particularmente en bebés prematuros y de muy bajo peso al nacer. 


\section{Epilepsia infantil diagnóstico, tratamiento y recomendación para padres}

Vol. 3, núm. 1., (2019)

Daniel Fernando Dick Paredes; Jenniffer Cristina Pazmiño Farfán; John Michael Franco

Orellana; Mayra Alexandra Bermeo Naranjo

- Identificación de cualquier terapia neuroprotectora que puede reducir o prevenir cualquier daño cerebral secundario después de un insulto primario inicial en la vida temprana.

- Reconocimiento temprano y tratamiento adecuado de meningitis, encefalitis y abscesos intracraneales, particularmente en bebés y niños pequeños.

- Mejor aceptación de las inmunizaciones infantiles para reducir tanto las encefalopatías agudas como las complicaciones tardías asociadas a estas enfermedades.

- Mejorar la reanimación de niños con lesión cerebral traumática y no traumática y la prevención del daño cerebral secundario.

- Identificación correcta y manejo agresivo del epiléptico relativamente raro pero importante encefalopatías (por ejemplo, el síndrome de West, Landau-Síndrome de Kleffner), que si no se reconoce puede causar potencialmente deterioro cognitivo irreversible.

- Evitar el uso inadecuado de AED y específicamente medicamentos que pueden exacerbar las convulsiones.

- Evitar el uso de múltiples AED y en alta dosis que pueden tener un efecto adverso sobre concentración y memoria a corto plazo que, incluso si transitoria, todavía puede reducir irreversiblemente el potencial educativo.

- Mejora pre, intra y postoperatoria. Acceso neuroquirúrgico y atención a los niños que requieren un tratamiento quirúrgico para su epilepsia. 


\section{Epilepsia infantil diagnóstico, tratamiento y recomendación para padres}

Vol. 3, núm. 1., (2019)

Daniel Fernando Dick Paredes; Jenniffer Cristina Pazmiño Farfán; John Michael Franco Orellana; Mayra Alexandra Bermeo Naranjo

- Identificación del niño (y de la familia, incluyendo a los hermanos) comprensión y percepciones de la epilepsia para prevenir cualquier adicional psicopatología dentro de la familia.

- Mayor conciencia, conocimiento y comprensión de la epilepsia: qué es y qué no es, entre todos los profesionales de la salud y la educación del público para reducir, si no eliminar, la estigmatización y prejuicio contra todas las personas con epilepsia.

\section{Conclusiones.}

Las convulsiones pediátricas y las epilepsias son emergencias que requieren un tratamiento temprano y efectivo. Todo el mundo es consciente de que, para todo esto, los resultados de los pacientes pueden mejorarse utilizando medicamentos antiepilépticos en la forma y dosis apropiada. Los estudios adicionales deben centrarse en el manejo de las convulsiones de un paciente pediátrico o epiléptico a través de la mejora del tratamiento teniendo en cuenta que la gestión de la vía aérea es una prioridad en pacientes pediátricos con esta condición; los niños con convulsiones febriles en la anamnesis deben ser evaluados a través del examen neurológico y el monitoreo del desarrollo mental, las causas de la fiebre deben siempre investigarse y tratarse, deben excluirse otras causas de convulsiones y la ansiedad de los padres debe ser controlada.

\section{Bibliografía.}

Berg, A. (2011). New concepts in classification of the epilepsies: Entering the 21st century. Epilepsia, 1058-1062.

Bolívar, J. (2015). Investigación Documental. México. Pax. 


\section{Epilepsia infantil diagnóstico, tratamiento y recomendación para padres}

Vol. 3, núm. 1., (2019)

Daniel Fernando Dick Paredes; Jenniffer Cristina Pazmiño Farfán; John Michael Franco

Orellana; Mayra Alexandra Bermeo Naranjo

Castro, J. (2016). Técnicas Documentales. México. Limusa.

Davila, A. (2015). Diccionario de terminos cientificos. Caracas: Oasis.

Guerrini, R. (2006). Epilepsy in children. Lancet.

Hauser, W., \& Banerjee, P. (2008). Epidemiology of Epilepsy in Children. Demos Medical Publishing, LLC.

Piña-Garza, E. F. (2013). La neurología pediátrica clínica de Fenichel: signos y enfoque de los sintomas. 1era Edicion. Elsevier Health Science, 396.

Sharma, A. (2013). Seizures and epilepsy in children. Indian J. Pediatr, 925-935.

Tamber, M., \& Mountz, J. (2012). Advances in the diagnosis and treatment of epilepsy. Seminars nuclear med, 371-386. 